\title{
Effectiveness of dementia follow-up care by memory clinics or general practitioners: randomised controlled trial
}

\section{Els J Meeuwsen scientific researcher in geriatrics ${ }^{1}$, René J F Melis senior researcher in geriatrics ${ }^{1}$, Geert C H M Van Der Aa geriatrician ${ }^{2}$, Gertie A M Golüke-Willemse geriatrician ${ }^{3}$, Benoit J M De Leest geriatrician ${ }^{4}$, Frank H J M Van Raak psychologist ${ }^{5}$, Carla J M Schölzel-Dorenbos geriatrician ${ }^{16}$, Desiree C M Verheijen geriatrician ${ }^{7}$, Frans R J Verhey professor of old age psychiatry and neuropsychiatry ${ }^{8}$, Marieke C Visser neurologist ${ }^{9}$, Claire A Wolfs senior researcher in psychiatry and psychology ${ }^{8}$, Eddy M M Adang senior researcher in efficiency studies ${ }^{10}$, Marcel G M Olde Rikkert professor in geriatrics ${ }^{1}$}

\begin{abstract}
'Department of Geriatrics/Alzheimer Centre Nijmegen, Radboud University Nijmegen Medical Centre, PO Box 9101, 6500 HB, Nijmegen, Netherlands; ${ }^{2}$ Department of Geriatrics, Catharina Hospital, 5602 ZA, Eindhoven, Netherlands; ${ }^{3}$ Department of Geriatrics, Rijnstate Hospital, 6800 TA, Arnhem, Netherlands; ${ }^{4}$ Department of Geriatrics, Elkerliek Hospital, 5700 AB, Helmond, Netherlands; ${ }^{5} \mathrm{GGZ}$ Oost-Brabant, Centre Land van Cuijk, 5830 AC, Boxmeer, Netherlands; ${ }^{6}$ Memory Clinic, Slingeland Hospital, 7000 AD, Doetinchem, Netherlands; ${ }^{7}$ Department of Geriatrics, Gelderse Vallei Hospital, 6710 HN, Ede, Netherlands; ${ }^{8}$ Department of Psychiatry and Neuropsychology/Alzheimer Centre Limburg, Maastricht University Medical Centre+, 6202 AZ, Maastricht, Netherlands; ${ }^{9}$ Department of Neurology/Alzheimer Centre Amsterdam, VU Medical Centre Amsterdam, 1007 MB, Amsterdam, Netherlands; ${ }^{10}$ Department of Epidemiology, Biostatistics and HTA, Radboud University Nijmegen Medical Centre
\end{abstract}

\begin{abstract}
Objective To examine the effectiveness of post-diagnosis dementia treatment and coordination of care by memory clinics compared with general practitioners.

Design Multicentre randomised controlled trial.

Setting Nine memory clinics and 159 general practitioners in the Netherlands.

Participants 175 patients with a new diagnosis of mild to moderate dementia living in the community and their informal caregivers.

Interventions Usual care provided by memory clinic or general practitioner.
\end{abstract}

Main outcome measures Caregiver rated quality of life of the patient measured with the quality of life in Alzheimer's disease instrument and self perceived burden of the informal caregiver measured with the sense of competence questionnaire (intention to treat analysis).

Results The quality of life of the patients in the memory clinic group was 0.5 (95\% confidence interval -0.7 to 1.6$)$ points higher than in the general practitioner group. Caregivers' burden was $2.4(-5.8$ to 1.0$)$ points lower in the memory clinic group than in the general practitioner group.

Conclusion No evidence was found that memory clinics were more effective than general practitioners with regard to post-diagnosis treatment and coordination care for patients with dementia. Without further evidence on the effectiveness of these modalities, other arguments, such as cost minimisation, patients' preferences, or regional health service planning, can determine which type of dementia care is offered.

Trial registration Clinical trials NCT00554047.

\section{Introduction}

Memory clinics used to focus on diagnosing dementia. However, they are increasingly involved in post-diagnosis treatment and coordination of care, especially since the introduction of anti-dementia drugs (cholinesterase inhibitors) in the 1990s. Previous research has indicated that memory clinics as diagnostic facilities are an efficient healthcare investment, ${ }^{1-4}$ but no direct scientific evidence shows the effectiveness of treatment and follow-up of dementia by memory clinics. ${ }^{56}$

A few years ago, Great Britain announced a national dementia strategy. ${ }^{7}$ The main goals of the strategy are improving public and professional awareness of dementia, enhancing early diagnosis and intervention for everyone, and providing higher quality care and support during all stages of dementia. ${ }^{8}{ }^{9}$ This national strategy intends to achieve its goals by providing easy 
access to services and support through establishing a nationwide network of multidisciplinary memory clinics. The implementation of this strategy has led to a widespread discussion on the justification of this approach. ${ }^{6}{ }^{10-14}$ The critics of this strategy question the effectiveness of the treatment and follow-up memory clinics provide after a diagnosis is made. ${ }^{15}$ The discussion about the best way to treat and provide care for patients with dementia is also a matter of debate in other countries. ${ }^{14} 1617$ To our knowledge, no randomised trials comparing the effectiveness of post-diagnosis dementia treatment and care coordination by memory clinics and general practitioners have been published. The PLASA study, which was recently conducted in France, compared a comprehensive specific care plan with usual dementia follow-up care in memory clinic settings. ${ }^{18}$ Furthermore, few studies have evaluated the effectiveness of memory clinics as a diagnostic setting. Wolfs and colleagues showed that in comparison with the usual diagnostic approach, an integrated multidisciplinary diagnostic approach for dementia was effective. ${ }^{4}$ Another study showed some beneficial effects of diagnosis of dementia at a memory clinic (as opposed to not being offered this service) on the health related quality of life of caregivers. ${ }^{19}$ However, the important question of what is the best way to organise post-diagnosis treatment and coordination of care for dementia remains unanswered. We designed this study to determine the effectiveness of post-diagnosis treatment and care coordination for patients with dementia and their caregivers by memory clinics compared with care provided by general practitioners.

\section{Methods}

\section{Study design}

This study (the AD-Euro Study), was a pragmatic multicentre randomised trial with 12 months' follow-up. Web based randomisation took place after baseline measurements. Participants (patient-caregiver pairs) were assigned for post-diagnosis dementia care to either the memory clinic or the general practitioner. Minimisation factors used for randomisation were patient's sex, caregiver's sex, patient's age, type of dementia, severity of dementia, the relationship between patient and caregiver, and centre. ${ }^{20}$ Details of the study design have been published elsewhere. ${ }^{21}$

\section{Participants}

From December 2007 until July 2009, nine Dutch memory clinics recruited participants after diagnostic investigation. Patients had to be newly diagnosed as having dementia meeting the criteria of the Diagnostic and Statistical Manual of Mental Disorders, fourth edition (DSM-IV), with a clinical dementia rating of $0.5,1$, or $2 .{ }^{22}$ Each patient had an informal caregiver, and patient and informal caregiver both gave informed consent before inclusion in the study. Patient-caregiver pairs were excluded when the patient lived in a nursing home, had a life expectancy of less than a year, or needed specific memory clinic care (for example, in the case of Creutzfeldt-Jakob disease) that could not be given by general practitioners.

\section{Intervention}

The interventions in this study consisted of usual care by either the memory clinic or the general practitioner. The memory clinic provided treatment and care coordination based on the specialist Dutch dementia guideline of the Dutch Institute for Healthcare Improvement. ${ }^{23}$ The main content of the intervention of the memory clinic was prescribing and guidance of anti-dementia drugs (cholinesterase inhibitors and memantine). Furthermore, they provided non-drug interventions-for example, occupational therapy, providing day structure, or referral to a nurse specialist, day care, or home care. Using the guidelines mentioned, both drug prescription/guidance and the non-drug interventions were delivered on a tailored basis.

Patient-caregiver pairs assigned to the general practitioner received post-diagnosis treatment and care provided by the general practitioner based on the Dutch general practice and homecare dementia guidelines. ${ }^{24}{ }^{25}$ As usual, the general practitioner received a discharge letter with advice about treatment after diagnostic investigation by the memory clinic. Contrary to the Dutch specialist guideline on dementia treatment, the general practice guideline states that the use of cholinesterase inhibitors is not recommended; however, several general practitioners did prescribe dementia drugs as part of the intervention. Most non-drug interventions available in memory clinic care are also available in general practitioner care and were also delivered on a tailored basis.

\section{Measurements}

After baseline measurements, follow-up measurements were made at six and 12 months. Research assistants, who were blinded to group allocation, made these measurements at the patient's home. At three and nine months, a short interview by telephone was conducted with the informal caregiver. An overview of the outcome measures and when they were used has been published elsewhere. ${ }^{21}$

\section{Primary outcome measures}

Primary outcome measures to establish effectiveness were the quality of life of the patient as rated by the caregiver, using the quality of life in Alzheimer's disease instrument (range 13-52; higher scores indicate a better quality of life), ${ }^{26}$ and self perceived caregiving burden of the informal caregiver, as measured by the sense of competence questionnaire (range 27-135; higher score reflects a greater sense of competence). ${ }^{27}$

\section{Secondary outcome measures}

Several secondary outcome measures in both patients and caregivers were assessed. To measure patients' depression, we used the geriatric depression scale, ${ }^{28}$ a short questionnaire validated in mild to moderate dementia. We measured behavioural disturbance by using the neuropsychiatric inventory in questionnaire format and the patient's functional performance by using the interview for deterioration in daily living activities in dementia scale. ${ }^{29}{ }^{30}$ Secondary outcome measures related to the caregiver were mood measured with the Centre for Epidemiologic Studies depression scale and anxiety measured with the state-trait anxiety inventory. ${ }^{31}{ }^{32} \mathrm{We}$ also used the Eysenck personality questionnaire to evaluate caregivers' personality and the Pearlin mastery scale to determine the amount of mastery (the extent to which life chances are seen as being under a person's own control in contrast to being fatalistically ruled). ${ }^{33}{ }^{34} \mathrm{We}$ measured emotional problems of the caregiver concerning the behaviour of the patient with the neuropsychiatric inventory in questionnaire format. ${ }^{29}$ To measure social support, we used the inventory for measuring social involvement. ${ }^{35}$

\section{Statistical analysis}

We used descriptive statistics to analyse baseline characteristics. We analysed effectiveness in two linear regression models on the quality of life in Alzheimer's disease instrument and sense of competence questionnaire, with the baseline value of the 
respective outcome measure and the minimisation factors added to the model as covariates. ${ }^{36} \mathrm{We}$ used an intention to treat analysis. ${ }^{37}$ As a minimal clinically important difference, we defined an average change in the quality of life in Alzheimer's disease instrument of 3 points on a scale ranging from 13 (very poor quality of life) to 52 (very good quality of life) and used a standard deviation of 6 in the sample size calculation. We judged an improvement of 3 points on this instrument to be clinically relevant, as this represents a change of wellbeing on one domain of quality of life from very poor to excellent. ${ }^{26}$ This change was also judged clinically relevant in an earlier intervention trial in dementia. ${ }^{26}{ }^{38}$ For the sense of competence questionnaire, we defined a minimal clinically important difference as a 5 point change on a scale from 27 to 135 (higher score reflects a greater sense of competence) and used a standard deviation of 8 in the sample size calculation. An improvement of 5 points was used in another trial on the effects of occupational therapy in Alzheimer's disease. ${ }^{39}$

We regarded memory clinic care as superior to general practitioner care when it was either superior on the quality of life in Alzheimer's disease instrument and non-inferior on the sense of competence questionnaire (or vice versa) or superior on both measures at 12 months' follow-up. We set the lower margin for non-inferiority at -1 for the quality of life in Alzheimer's disease instrument and at -1.5 for the sense of competence questionnaire. We calculated the sample size to be 220 patients, accounting for a $30 \%$ attrition rate equally distributed over both arms. With $\alpha=0.05$ and an expected treatment difference in favour of memory clinic care of 3 points on the quality of life in Alzheimer's disease instrument and 5 points on the sense of competence questionnaire, the power was 0.98 for showing superiority on at least one of the outcome measures and non-inferiority on the other. The power was 0.84 for showing superiority on both outcome measures. We did not replace missing values, given that the attrition rate was modest. ${ }^{21}$ We did sensitivity analyses for the effect of missing data on the results of the primary analyses by using the last observation carried forward method and by replacing the missing value with the mean of the other group. ${ }^{40}$ We used Microsoft Office Excel 2007 and SPSS 16.0.01 (release 16.0.2) to do the statistical analyses.

\section{Results}

We included 175 patient-caregiver pairs in the study; 87 were randomly assigned to the memory clinic group and 88 to the general practitioner group (figure $\Downarrow$ ). Baseline characteristics of patients and caregivers were similar between the two groups (table $1 \Downarrow)$. Most of the patients $(61 \%, \mathrm{n}=106)$ and caregivers $(70 \%, \mathrm{n}=123)$ were female. The average age of the patients was 78.1 (SD 5.7) years, and caregivers were on average 63.5 (13.1) years old. More than half of the caregivers $(54 \%, n=94)$ were partners, either married or living together with the patient, and $41 \%(n=72)$ of the caregivers were daughters or sons (or daughters or sons in law). Most of the patients $(60 \%, n=105)$ had Alzheimer's disease; in $84 \%(n=147)$ of the patients, the severity of the dementia was very mild to mild (clinical dementia rating 0.5 and 1). Cognition of the patients, measured with the mini-mental state examination (range 0-30; higher score indicates better $\operatorname{cognition}^{41}$ ) at baseline was on average 22.7 (SD 3.9).

Of the 175 pairs included in the study, 22 pairs dropped out during the follow-up period-13 pairs in the general practitioner group and nine pairs in the memory clinic group (figure $\Downarrow$ ). Four patients (three in the memory clinic group) and two caregivers (both in the general practitioner group) died during the follow-up phase. Eleven pairs (four in the memory clinic group) dropped out because they considered further participation to be too burdensome. Two caregivers (one in each group) contracted a serious illness and dropped out. One caregiver in the memory clinic group did not fill out the questionnaires, and one caregiver in the general practitioner group was not present during the measurements without giving any reasons. Finally, one diagnosis of dementia in a patient in the general practitioner group was changed just after inclusion.

\section{Outcomes}

Out of the 175 patient-caregiver pairs, 153 pairs (75 in the general practitioner group and 78 in the memory clinic group) were evaluated for the primary effectiveness outcomes (quality of life in Alzheimer's disease instrument and sense of competence questionnaire) at 12 months' follow-up. The patients in the memory clinic group scored 0.5 (95\% confidence interval -0.7 to 1.6) points higher on the quality of life in Alzheimer's disease instrument than did those in the general practitioner group. Caregivers in the memory clinic group scored 2.4 (-5.8 to 1.0) points lower on the sense of competence questionnaire than did those in the general practitioner group (table $2 \Downarrow$ ). None of the differences was statistically significant. This was also the case at six months' follow-up (table $2 \Downarrow$ ). The results of the sensitivity analyses for the primary outcome measures replacing the missing values by using last observation carried forward and with the mean of the other group were comparable to the results presented here.

After 12 months of follow-up, we found no statistically significant difference between treatment arms for the secondary outcome measures of the patients (table $2 \Downarrow$ ). Three secondary outcome measures of caregivers showed statistically significant differences between the two study groups in favour of the general practitioner group (table $2 \Downarrow$ ). These were two scales of the state-trait anxiety inventory, measuring anxiety in general (anxiety trait) and anxiety at the moment of measuring (anxiety state), and the Centre for Epidemiologic Studies depression scale, which measured depressive symptoms of the caregiver. Anxiety trait was $2.1(0.24$ to 4.03$)$ points higher, anxiety state was 2.4 ( 0.35 to 4.36 ) points higher, and depression was 2.1 ( 0.15 to 4.02 ) points higher for the memory clinic group compared with the general practitioner group. However, the average scores on these instruments for the two groups were well below the cut-offs for relevant clinical symptoms of anxiety or depression. ${ }^{31} 32$ On the outcome measures state-trait anxiety inventory and Centre for Epidemiologic Studies depression scale, we found differences in baseline scores between participants who completed the study and those who dropped out. Also, differences existed between the two study arms: dropouts in the general practitioner arm had worse mean baseline values for anxiety trait, anxiety state, and depression (41.9, 44.2, and 11.7), than did dropouts in the memory clinic arm, who had values of 35.5, 35.2, and 9.3 $(\mathrm{P}<0.05)$. Baseline and 12 month follow-up scores on each of these instruments were highly correlated, with correlation coefficients of $0.79,0.69$, and 0.64 . To better understand how these differences may have influenced the secondary outcome results, we did the analysis of covariance again with last observation carried forward for the dropouts. On both the state-trait anxiety inventory and the Centre for Epidemiologic Studies depression scale, the differences between the two study groups got smaller and lost statistical significance. At six months' follow-up, two secondary outcome measures of the caregiver, anxiety state and inventory for measuring social involvement, showed significant differences between the groups 
in favour of the general practitioners group (table $2 \Downarrow$ ). Again, participants who dropped out in the general practitioner group at six months' follow-up had a worse mean baseline value for anxiety state than did dropouts in the memory clinic group ( 43.8 $v 37.5)$. We found no difference in the mean baseline value between completers and dropouts on the inventory for measuring social involvement.

\section{Discussion}

We found no evidence of a difference in effectiveness between memory clinics and general practitioners with regard to post-diagnosis treatment and coordination of care for patients with dementia. The analysis suggests that being guided by a general practitioner had a small positive effect on anxiety or mood of the caregiver (secondary outcome measures), but this may be the effect of selective loss to follow-up.

\section{Comparison with other studies}

Comparison of our results with others is difficult, because of the lack of similar studies. The PLASA study looked at effectiveness of a specific care plan provided by memory clinics compared with regular care provided by memory clinics. ${ }^{18}$ They saw no additional positive effect on functional decline in patients with Alzheimer's disease. Although the primary outcome in the PLASA study was functional decline, this lack of additional effect seems to correspond with our results. The study of Wolfs et al showed that, in comparison with usual care, an integrated multidisciplinary diagnostic approach to dementia in a memory clinic setting increased the health related quality of life of dementia patients and was cost effective. ${ }^{3}$ Compared with our study, their patients' cognition was worse and the patients had a much lower mean quality adjusted life year value, indicating that our study included a population with better cognition and better quality of life. Furthermore, a simple association may not exist between outcomes relevant to patients such as health related quality of life and discrete areas of function such as cognition and behaviour. Moreover, the responsiveness of outcome measures relevant to patients may be debated, specifically in dementia patients. ${ }^{42}$ Therefore, next to the primary outcome measure, the quality of life in Alzheimer's disease instrument, we also evaluated the two types of care for their effects on clinically relevant outcome measures such as function, cognition, and behaviour, but we were unable to show superiority for memory clinic care on these measures. Moreover, because of the importance of the burden on caregivers, we used the sense of competence questionnaire as a primary outcome measure. This turned out to be highly responsive in a randomised controlled trial of occupational therapy in the same population of dementia patients. ${ }^{39} \mathrm{We}$ found no indications for a difference between the memory clinic and general practitioner groups on the sense of competence questionnaire.

\section{Strengths and limitations}

By using a randomised clinical trial, we ensured a robust study design. The participation of nine different memory clinics, based in different settings (university hospital, general hospital, old age psychiatry), enhanced the generalisability of our study. However, we recruited participants only from memory clinics and not from general practices, which means that the results may not be representative for all patients with mild to moderate dementia in the general population, although a considerable proportion $(27 \%)$ of all cases of dementia are diagnosed at a memory clinic in the Netherlands. ${ }^{43}$ Recruiting patients through the memory clinics ensured a thorough and consistent diagnosis.
On the other hand, differences in healthcare systems between countries make generalisability more difficult. Variability in dementia care exists within the Netherlands as well as between different countries, possibly owing to the rapid increase in the number of memory clinics recently and the many developments in dementia care. However, quality indicators for both general practitioners and memory clinics are now being implemented in different European countries, which will hopefully improve comparability of dementia care in the future..$^{44-46}$

Two other limitations could have affected the outcomes of this study. Dementia is a disease that progresses over years, so an extended follow-up lasting several years would be preferable to the relatively short 12 month period we used. In addition, the type of intervention under study did not allow us to use double blind procedures. However, as our study was a pragmatic trial, ${ }^{21}$ biases as a result of not using double blind procedures are generally not viewed as detrimental but accepted as part of physicians' and patients' responses to treatment and included in the overall assessment. ${ }^{47}$ In pragmatic approaches, the treatment response is the total difference between two treatments, which includes both treatment and placebo effects, as this will best reflect the likely clinical response in practice. ${ }^{47} 48$

\section{Implications and conclusions}

With the results of our study, we provide empirical data on differences in effectiveness between memory clinics and general practitioners. These data can now fuel the ongoing debate on which type of post-diagnosis treatment and follow-up is best for which patients. The debate that followed the appearance of the UK national dementia strategy (NDS), has once more stressed the need to continue to compare different strategies of dementia care and to continue to increase the evidence base: "NDS is not a beginning but a point in time in the progressive improvement of services for people with dementia and their families. . Strengthening the potential of primary care and including it in collaborative systems will be the best way to use our new knowledge."15

The AD-Euro study adds important data, which seem to point to a lack of difference in effectiveness between memory clinics and general practitioners in the treatment and coordination of care for patients with dementia. Other factors can and should thus determine the preference for one provider over the other-for example, patients' and caregivers' preferences or regional or national planning to concentrate specific services. Cost minimisation may also be a strong argument if quality of care can be shown to be guaranteed. Further empirical data are needed before we will be able to press this point. A different and potentially very promising direction would be to start to integrate the resources of memory clinics and general practitioners and to combine their knowledge and experience to build truly integrated dementia care that may optimally serve the increasing number of patients with dementia in the future.

We thank all participants for their contribution. We also thank the research assistants for carrying out all the measurements and the staff of the memory clinics for their participation.

Contributors: GCHMVDA, GAMG-W, BJMDL, FHJMVR, CJMS-D, DCMV, FVRJ, MCV, CAW, and MGMOR recruited the participants. EJM, RJFM, and EMMA analysed the data and, with and MGMOR, interpreted the data and wrote the manuscript. All authors approved the final manuscript. MGMOR is the guarantor.

Funding: This work was supported by ZonMw (Netherlands Organization for Health Research and Development; grant no 945-07-703) and by the Radboud University Nijmegen Medical Centre. 


\section{What is already known on this topic}

The number of memory clinics has increased rapidly over the past decades

Memory clinics have been shown to be effective as diagnostic facilities

\section{What this study adds}

No evidence was found of a difference in effectiveness between memory clinics and general practitioners in treating and coordinating care for patients with dementia

Patients' and caregivers' preferences, cost minimisation, or a policy of concentrating specific services may determine the preference for memory clinic or general practitioner for post-diagnosis care

Competing interests: All authors have completed the Unified Competing Interest form at www.icmje.org/coi_disclosure.pdf (available on request from the corresponding author) and declare: no support from any organisation for the submitted work; no financial relationships with any organisations that might have an interest in the submitted work in the previous three years; no other relationships or activities that could appear to have influenced the submitted work.

Ethical approval: The study was approved by the Medical Ethics Committee of the Radboud University Nijmegen Medical Centre.

Data sharing: The dataset is available from the corresponding author at e.meeuwsen@ger.umcn.nl. Participants' consent was not obtained, but the data presented are anonymised and risk of identification is low.

1 Geldmacher DS. Cost-effective recognition and diagnosis of dementia. Semin Neurol 2002;22:63-70

2 Van Crevel H, van Gool WA, Walstra GJ. Early diagnosis of dementia: which tests are indicated? What are their costs? J Neurol 1999;246:73-8.

3 Wolfs CA, Dirksen CD, Kessels A, Severens JL, Verhey FR. Economic evaluation of an integrated diagnostic approach for psychogeriatric patients: results of a randomized controlled trial. Arch Gen Psychiatry 2009;66:313-23.

4 Wolfs CA, Kessels A, Dirksen CD, Severens JL, Verhey FR. Integrated multidisciplinary diagnostic approach for dementia care: randomised controlled trial. Br J Psychiatry 2008;192:300-5.

5 Passmore AP, Craig DA. The future of memory clinics. Psychiatric Bulletin 2004;28:375-7.

6 Melis RJ, Meeuwsen EJ, Parker SG, Olde Rikkert MG. Are memory clinics effective? The odds are in favour of their benefit, but conclusive evidence is not yet available. $J R$ Soc Med 2009;102:456-7.

7 Department of Health. Living well with dementia: a national dementia strategy. Department of Health, 2009.

8 Jolley D, Benbow SM, Grizzell M. Memory clinics. Postgrad Med J 2006;82:199-206.

9 Banerjee S. Living well with dementia-development of the national dementia strategy for England. Int J Geriatr Psychiatry 2010;25:917-22.

10 Burns A, Robert P. The national dementia strategy in England. BMJ 2009;338:b931.

11 Coombes $\mathrm{R}$. Evidence lacking for memory clinics to tackle dementia, say critics. $B M J$ 2009;338:b550.

12 Hean S, Nojeed N, Warr J. Developing an integrated memory assessment and support service for people with dementia. J Psychiatr Ment Health Nurs 2011;18:81-8.

13 Manthorpe J. National dementia strategy. Br J Gen Pract 2010;60:373.

14 Cahill S. Developing a national dementia strategy for Ireland. Int J Geriatr Psychiatry 2010;25:912-6.

15 Greaves I, Jolley D. National dementia strategy: well intentioned-but how well founded and how well directed? Br J Gen Pract 2010;60:193-8.

16 Connelly P. Scottish dementia strategy editorial. Int J Geriatr Psychiatry 2010;25:908-11.

17 Eriksson S. Developments in dementia strategy. Int J Geriatr Psychiatry 2010;25:885-6.

18 Nourhashemi F, Andrieu S, Gillette-Guyonnet S, Giraudeau B, Cantet C, Coley N, et al. Effectiveness of a specific care plan in patients with Alzheimer's disease: cluster randomised trial (PLASA study). BMJ 2010;340:c2466.

19 LoGiudice D, Waltrowicz W, Brown K, Burrows C, Ames D, Flicker L. Do memory clinics improve the quality of life of carers? A randomized pilot trial. Int J Geriatr Psychiatry 1999;14:626-32.

20 Altman DG, Bland JM. Treatment allocation by minimisation. BMJ 2005;330:843

21 Meeuwsen EJ, Melis RJ, Adang EM, Goluke-Willemse GA, Krabbe PF, de Leest BJ, et al. Cost-effectiveness of post-diagnosis treatment in dementia coordinated by multidisciplinary memory clinics in comparison to treatment coordinated by genera practitioners: an example of a pragmatic trial. J Nutr Health Aging 2009;13:242-8.

22 Morris JC. The clinical dementia rating (CDR): current version and scoring rules. Neurology 1993:43:2412-4

$23 \mathrm{CBO}$ richtlijn. Diagnostiek en medicamenteuze behandeling van dementie. Nederlandse Vereniging voor Klinische Geriatrie, ed. Van Zuiden Communications BV, 2005.

24 Nederlands Huisartsen Genootschap. NHG-Standaard Dementie M21, 2003.
25 Boomsma LJ, De Bont M, Engelsman C, Gussekloo J, Hartman C, Persoon A, et al. Landelijke eerstelijns samenwerkings afspraak dementie [Dutch]. Huisarts Wet 2005;48:124-6.

26 Logsdon RG, Gibbons LE, McCurry SM, Teri L. Assessing quality of life in older adults with cognitive impairment. Psychosom Med 2002;64:510-9.

27 Vernooii-Dassen MJ, Persoon JM, Felling AJ. Predictors of sense of competence in caregivers of demented persons. Soc Sci Med 1996;43:41-9.

28 Yesavage JA, Heikh JI. Geriatric depression scale (GDS) recent evidence and development of a shorter violence. Clin Gerontol 1986;5:165-73.

29 Kaufer DI, Cummings JL, Ketchel P, Smith V, MacMillan A, Shelley T, et al. Validation of the NPI-Q, a brief clinical form of the neuropsychiatric inventory. J Neuropsychiatry Clin Neurosci 2000:12:233-9.

30 Teunisse S, Derix MM. Measurement of activities of daily living in patients with dementia living at home: development of a questionnaire [Dutch]. Tijdschr Gerontol Geriat 1991;22:53-9.

31 Radloff LS. The CES-D scale: a self-report depression scale for research in the general population. Appl Psychol Meas 1977;1:385-401.

32 Spielberger CD, Gorsuch RL, Lushene RE. STAI manual for the state-trait anxiety inventory. Consulting Psychologists Press, 1970

33 Eysenck SB, Eysenck HJ. The questionnaire measurement of psychoticism. Psychol Med 1972;2:50-55.

34 Pearlin LI, Schooler C. The structure of coping. J Health Soc Behav 1978;19:2-21.

35 Dam van-Baggen CMJ, Huiskes CJAE, Kraaimaat FW. Inventarisatielijst sociale betrokkenheid: ISB (inventory for measuring social involvement). Academic Hospital Utrecht, 1986.

36 Borm GF, Fransen J, Lemmens WA. A simple sample size formula for analysis of covariance in randomized clinical trials. J Clin Epidemiol 2007;60:1234-8.

37 Hollis $\mathrm{S}$, Campbell F. What is meant by intention to treat analysis? Survey of published randomised controlled trials. BMJ 1999;319:670-4.

38 Spector A, Thorgrimsen L, Woods B, Royan L, Davies S, Butterworth M, et al. Efficacy of an evidence-based cognitive stimulation therapy programme for people with dementia: randomised controlled trial. Br J Psychiatry 2003;183:248-54

39 Graff MJ, Vernooij-Dassen MJ, Thijssen M, Dekker J, Hoefnagels WH, Rikkert MG Community based occupational therapy for patients with dementia and their care givers: randomised controlled trial. BMJ 2006;333:1196.

40 Unnebrink K, Windeler J. Intention-to-treat: methods for dealing with missing values in clinical trials of progressively deteriorating diseases. Stat Med 2001;20:3931-46.

41 Folstein MF, Folstein SE, McHugh PR. Mini-mental state-practical method for grading cognitive state of patients for clinician. J Psychiatr Res 1975;12:189-98.

42 Banerjee S, Samsi K, Petrie CD, Alvir J, Treglia M, Schwam EM, et al. What do we know about quality of life in dementia? A review of the emerging evidence on the predictive and explanatory value of disease specific measures of health related quality of life in people with dementia. Int J Geriatr Psychiatry 2009:24:15-24.

43 Ramakers IH, Verhey FR. Development of memory clinics in the Netherlands: 1998 to 2009. Aging Ment Health 2011;15:34-9.

44 Draskovic I, Vernooij-Dassen M, Verhey F, Scheltens P, Rikkert MO. Development of quality indicators for memory clinics. Int J Geriatr Psychiatry 2008;23:119-28.

45 Perry M, Draskovic I, van AT, van EM, Lucassen P, Vernooij-Dassen M, et al. Development and validation of quality indicators for dementia diagnosis and management in a primary care setting 4. J Am Geriatr Soc 2010;58:557-63.

46 Doncaster E, McGeorge M, Orrell M. Developing and implementing quality standards for memory services: the memory services national accreditation programme (MSNAP) 1 . Aging Ment Health 2011;15:23-33.

47 Roland M, Torgerson DJ. Understanding controlled trials: what are pragmatic trials? BMJ 1998;316:285.

48 Ware $\mathrm{JH}$, Hamel MB. Pragmatic trials—guides to better patient care? N Engl J Med 2011:364:1685-7.

\section{Accepted: 26 March 2012}

\section{Cite this as: BMJ 2012;344:e3086}

This is an open-access article distributed under the terms of the Creative Commons Attribution Non-commercial License, which permits use, distribution, and reproduction in any medium, provided the original work is properly cited, the use is non commercial and is otherwise in compliance with the license. See: http://creativecommons.org/licenses/by$\mathrm{nc} / 2.0 /$ and http://creativecommons.org/licenses/by-nc/2.0/legalcode. 


\section{Tables}

Table 1| Baseline characteristics of participants in memory clinic (MC) group and general practitioner (GP) group. Values are numbers (percentages) unless stated otherwise

\begin{tabular}{|c|c|c|c|c|}
\hline \multirow[b]{2}{*}{ Characteristics } & \multicolumn{2}{|c|}{ MC group ( $n=87$ ) } & \multicolumn{2}{|c|}{ GP group $(n=88)$} \\
\hline & Patient & Caregiver & Patient & Caregiver \\
\hline Female & $54(62)$ & $62(71)$ & $52(59)$ & $61(69)$ \\
\hline Mean (SD) age (years) & $78.2(6.2)$ & $63.2(13.4)$ & $77.9(5.2)$ & $63.9(12.9)$ \\
\hline \multicolumn{5}{|l|}{ Type of dementia: } \\
\hline Alzheimer's disease & $53(61)$ & - & $52(59)$ & - \\
\hline Vascular dementia & $9(10)$ & - & $6(7)$ & - \\
\hline Mixed/other & $25(29)$ & - & $30(34)$ & - \\
\hline \multicolumn{5}{|l|}{ Severity of dementia: } \\
\hline CDR 0.5 & $3(3)$ & - & $5(6)$ & - \\
\hline CDR 1 & $70(80)$ & - & $69(78)$ & - \\
\hline $\mathrm{CDR} 2$ & $14(16)$ & - & $14(16)$ & - \\
\hline \multicolumn{5}{|l|}{ Relationship with caregiver: } \\
\hline Partner & $46(53)$ & - & $48(55)$ & - \\
\hline Child (in law) & $36(41)$ & - & $36(41)$ & - \\
\hline Other & $5(6)$ & - & $4(5)$ & - \\
\hline \multicolumn{5}{|l|}{ Education,: } \\
\hline Low & $31(36)$ & $10(11)$ & $30(34)$ & $7(8)$ \\
\hline Middle & $35(40)$ & $52(60)$ & $36(41)$ & $56(64)$ \\
\hline High & $20(23)$ & $25(29)$ & $21(24)$ & $24(27)$ \\
\hline \multicolumn{5}{|l|}{ Mean (SD) scores: } \\
\hline $\mathrm{SCQ}$ & - & $107.9(15.0)$ & - & $105.1(13.7)$ \\
\hline $\begin{array}{l}\text { QoL-AD patient, as rated by } \\
\text { caregiver }\end{array}$ & $30.6(5.2)$ & - & $30.7(4.7)$ & - \\
\hline CIRS G & $9.2(4.4)$ & - & $8.8(4.6)$ & - \\
\hline MMSE & $22.7(3.6)$ & - & $22.7(4.2)$ & - \\
\hline GDS & $2.6(2.5)$ & - & $2.6(2.3)$ & - \\
\hline IDDD help needed & $13.8(8.8)$ & - & $13.4(9.1)$ & - \\
\hline IDDD take initiative & $13.2(7.7)$ & - & $12.3(6.8)$ & - \\
\hline NPI behaviour & $7.1(6.1)$ & - & $7.7(6.2)$ & - \\
\hline QoL-AD & $36.1(4.2)$ & $37.6(3.6)$ & $36.2(4.4)$ & $38.5(4.7)$ \\
\hline CES D & - & $9.3(7.6)$ & - & $9.8(7.6)$ \\
\hline NPI emotional & - & $9.1(9.4)$ & - & $10.3(8.8)$ \\
\hline STAl trait & - & $34.0(10.2)$ & - & $34.6(9.1)$ \\
\hline STAI state & - & $34.9(9.7)$ & - & $36.5(9.3)$ \\
\hline $\mathrm{EPQ}$ & - & $2.6(3.2)$ & - & $3.0(2.9)$ \\
\hline PMS & - & $16.5(4.0)$ & - & $16.7(4.7)$ \\
\hline ISB & - & $12.2(3.0)$ & - & $12.3(3.2)$ \\
\hline
\end{tabular}

$\mathrm{CDR}=$ clinical dementia rating scale (range 0-3; higher score indicates more severe dementia); CES D=Center for Epidemiologic Studies depression scale (range 0-60; higher score indicates more depressive); CIRS G=cumulative illness rating scale for geriatrics (range 0-56; higher score indicates more comorbidity); $\mathrm{EPQ}=$ Eysenck personality questionnaire, short version, neuroticism (range 0-12; higher score indicates more worried); GDS=geriatric depression scale (range 0-15; higher score indicates more depressive); IDDD=interview for deterioration in daily living activities in dementia (range for "help needed" $0-44$; range for "take initiative" 0-36; higher score indicates more help needed or less initiative taken); ISB=inventory for measuring social involvement (range 4-16; higher score indicates more social support); MMSE=mini-mental state examination (range 0-30; higher score indicates better cognition); NPI behaviour=neuropsychiatric inventory in questionnaire format (range 0-36; higher score indicates more behavioural disturbance); NPI emotional= neuropsychiatric inventory in questionnaire format; range 0-60; higher score indicates more emotional problems); PMS=Pearlin mastery scale (range 7-35; higher score indicates less own control; QoL-AD=quality of life 
Table 1 (continued)

\begin{tabular}{llll} 
& \multicolumn{2}{c}{ MC group $(n=87)$} & \multicolumn{2}{c}{ GP group $(n=88)$} & Patient \\
\cline { 2 - 3 } Characteristics & Patient & Caregiver & Caregiver
\end{tabular}

in Alzheimer's disease (range 13-52; higher score indicates better quality of life); SCQ=sense of competence questionnaire (range 27-135; higher score reflects greater sense of competence); STAl=state-trait anxiety inventory (range 20-80; higher score indicates more anxiety. 
Table 2| Analysis of covariance for difference between memory clinic (MC) and general practitioner (GP) groups for primary and secondary outcome measures at 6 and 12 months' follow-up

\begin{tabular}{|c|c|c|c|c|c|c|}
\hline & \multicolumn{3}{|c|}{6 months' follow-up } & \multicolumn{3}{|c|}{12 months' follow-up } \\
\hline & $\begin{array}{c}\text { Difference }(95 \% \mathrm{Cl}) \\
\text { between } \mathrm{MC} \text { and } \mathrm{GP}^{*}\end{array}$ & $P$ value & No & $\begin{array}{c}\text { Difference (95\% } \mathrm{Cl}) \\
\text { between } \mathrm{MC} \text { and } \mathrm{GP}^{*}\end{array}$ & $P$ value & No \\
\hline \multicolumn{7}{|l|}{ Patients' outcomes } \\
\hline $\begin{array}{l}\text { QoL-AD patient, as } \\
\text { rated by caregiver } \dagger\end{array}$ & $0.58(-0.57$ to 1.73$)$ & 0.32 & 153 & $0.49(-0.66$ to 1.63$)$ & 0.40 & 153 \\
\hline NPI behaviour & $0.63(-0.81$ to 2.06$)$ & 0.39 & 154 & $1.13(-0.51$ to 2.77$)$ & 0.18 & 152 \\
\hline IDDD help needed & $1.30(-1.08$ to 3.69$)$ & 0.28 & 151 & $0.66(-1.88$ to 3.20$)$ & 0.61 & 148 \\
\hline IDDD take initiative & $0.44(-1.38$ to 2.26$)$ & 0.64 & 153 & $1.69(-0.18$ to 3.56$)$ & 0.08 & 152 \\
\hline GDS & $-0.03(-0.56$ to 0.50$)$ & 0.91 & 150 & $0.25(-0.36$ to 0.86$)$ & 0.41 & 139 \\
\hline QoL-AD patient & $0.19(-0.74$ to 1.13$)$ & 0.68 & 153 & $0.25(-0.76$ to 1.23$)$ & 0.62 & 145 \\
\hline \multicolumn{7}{|c|}{ Caregivers' outcomes } \\
\hline SCQ† & $-0.93(-3.75$ to 1.89$)$ & 0.52 & 156 & $-2.43(-5.82$ to 0.96$)$ & 0.16 & 153 \\
\hline QoL-AD caregiver & $-0.20(-1.07$ to 0.66$)$ & 0.64 & 154 & $0.17(-0.70$ to 1.04$)$ & 0.70 & 154 \\
\hline CES D & $0.05(-2.04$ to 2.13$)$ & 0.96 & 153 & $2.09(0.15$ to 4.02$)$ & 0.04 & 151 \\
\hline ISB & $-0.84(-1.54$ to -0.14$)$ & 0.02 & 154 & $-0.29(-0.97$ to 0.78$)$ & 0.39 & 151 \\
\hline NPI emotional & $1.29(-0.77$ to 3.35$)$ & 0.22 & 153 & $1.43(-0.94$ to 3.80$)$ & 0.24 & 152 \\
\hline EPQ & - & - & - & $0.68(-0.01$ to 1.36$)$ & 0.05 & 151 \\
\hline STAI trait & - & - & - & $2.14(0.24$ to 4.03$)$ & 0.03 & 152 \\
\hline STAl state & 3.55 (1.29 to 5.81$)$ & 0.002 & 151 & 2.35 (0.35 to 4.36$)$ & 0.02 & 151 \\
\hline PMS & $-0.03(-1.17$ to 1.10$)$ & 0.95 & 152 & $0.65(-0.51$ to 1.80$)$ & 0.27 & 152 \\
\hline
\end{tabular}

CES $D=$ Center for Epidemiologic Studies depression scale (range 0-60; higher score indicates more depressive); CIRS G=cumulative illness rating scale for geriatrics (range 0-56; higher score indicates more comorbidity); EPQ=Eysenck personality questionnaire, short version, neuroticism (range 0-12; higher score indicates more worried; GDS=geriatric depression scale (range 0-15; higher score indicates more depressive); IDDD=interview for deterioration in daily living activities in dementia (range for "help needed" 0-44; range for "take initiative" 0-36; higher score indicates more help needed or less initiative taken); ISB=inventory for measuring social involvement (range 4-16; higher score indicates more social support); MMSE=mini-mental state examination (range 0-30; higher score indicates better cognition); NPI behaviour=neuropsychiatric inventory in questionnaire format (range 0-36; higher score indicates more behavioural disturbance); $\mathrm{NPI}$ emotional= neuropsychiatric inventory in questionnaire format; range 0-60; higher score indicates more emotional problems); PMS=Pearlin mastery scale (range 7-35; higher score indicates less own control; QoL-AD=quality of life in Alzheimer's disease (range 13-52; higher score indicates better quality of life); $\mathrm{SCQ}=$ sense of competence questionnaire (range 27-135; higher score reflects greater sense of competence); STAl=state-trait anxiety inventory (range 20-80; higher score indicates more anxiety.

*Adjusted for type of dementia, severity of dementia, patient's sex, patient's age, caregiver's sex, relationship of patient to caregiver, memory clinic involved, and baseline measurement of relevant outcome.

†Primary outcome measures.

¥Not measured at 6 months' follow-up. 


\section{Figure}

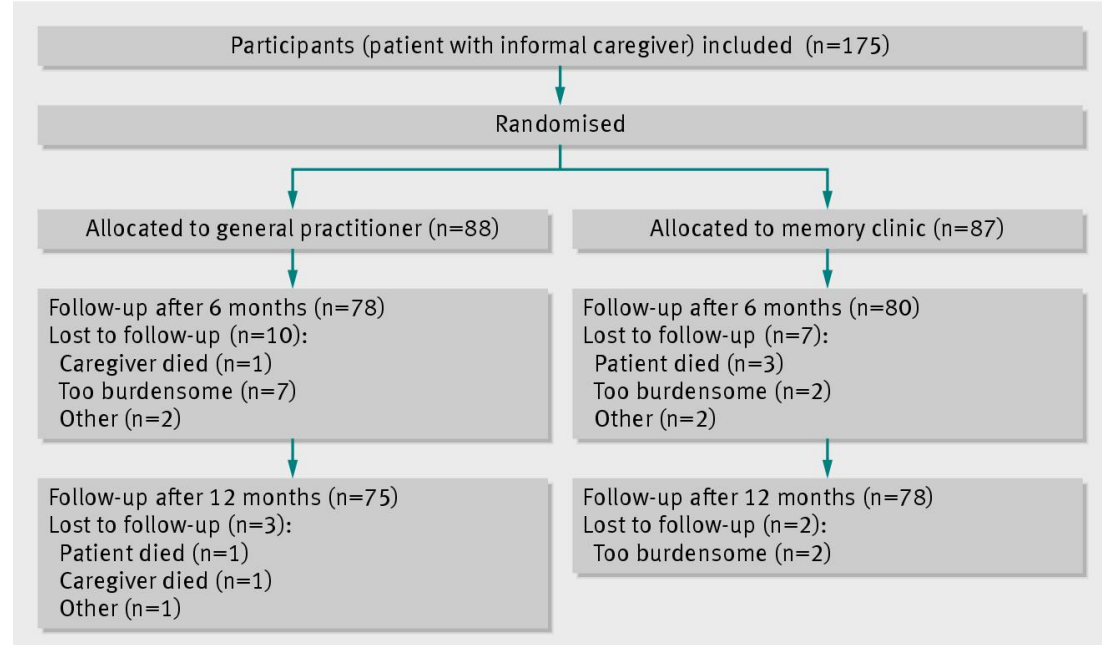

Flow diagram of study 